\title{
PROSPECT OF STUDYING HARD X- AND GAMMA-RAYS FROM TYPE Ia SUPERNOVAE
}

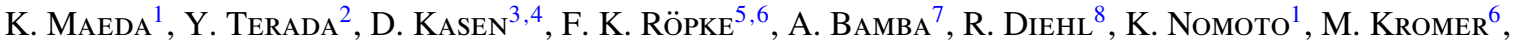

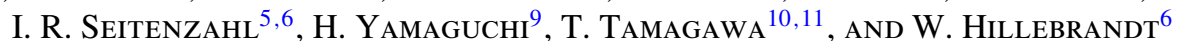 \\ ${ }^{1}$ Kavli Institute for the Physics and Mathematics of the Universe (Kavli-IPMU), Todai Institutes for Advanced Study (TODIAS), \\ University of Tokyo, 5-1-5 Kashiwanoha, Kashiwa, Chiba 277-8583, Japan; keiichi.maeda@ipmu.jp \\ ${ }^{2}$ Department of Physics, Saitama University, 255 Shimo-Okubo, Sakura-ku, Saitama 338-8570, Japan \\ ${ }^{3}$ Department of Physics, University of California at Berkeley, 366 LeConte, Berkeley, CA 94720, USA \\ ${ }^{4}$ Nuclear Science Division, Lawrence Berkeley National Laboratory, Berkeley, CA 94720, USA \\ ${ }^{5}$ Institut für Theoretische Physik und Astrophysik, Universität Würzburg, Emil-Fischer-Straße 31, D-97074 Würzburg, Germany \\ ${ }^{6}$ Max-Planck-Institut für Astrophysik, Karl-Schwarzschild-Straße 1, D-85741 Garching, Germany \\ ${ }^{7}$ Department of Physics and Mathematics, College of Science and Engineering, Aoyama Gakuin University, \\ 5-10-1 Fuchinobe, Chuo-ku, Sagamihara, Kanagawa 252-5258, Japan \\ ${ }^{8}$ Max-Planck-Institut für Extraterrestrische Physik, Giessenbachstraße 1, D-85748 Garching, Germany \\ ${ }^{9}$ Harvard-Smithsonian Center for Astrophysics, 60 Garden Street, Cambridge, MA 02138, USA \\ ${ }^{10}$ RIKEN (Institute of Physical and Chemical Research), 2-1 Hirosawa, Wako, Saitama 351-0198, Japan \\ ${ }^{11}$ Department of Physics, Tokyo University of Science, 1-3 Kagurazaka, Shinjuku-ku, Tokyo 162-8601, Japan \\ Received 2011 November 29; accepted 2012 October 9; published 2012 November 2
}

\begin{abstract}
We perform multi-dimensional, time-dependent radiation transfer simulations for hard X-ray and $\gamma$-ray emissions, following radioactive decays of ${ }^{56} \mathrm{Ni}$ and ${ }^{56} \mathrm{Co}$, for two-dimensional delayed-detonation models of Type Ia supernovae (SNe Ia). The synthetic spectra and light curves are compared with the sensitivities of current and future observatories for an exposure time of $10^{6} \mathrm{~s}$. The non-detection of the $\gamma$-ray signal from SN $2011 \mathrm{fe}$ at $6.4 \mathrm{Mpc}$ by SPI on board INTEGRAL places an upper limit on the mass of ${ }^{56} \mathrm{Ni}$ of $\lesssim 1.0 M_{\odot}$, independently from observations in any other wavelengths. Signals from the newly formed radioactive species have not yet been convincingly measured from any SN Ia, but future X-ray and $\gamma$-ray missions are expected to deepen the observable horizon to provide high energy emission data for a significant SN Ia sample. We predict that the hard X-ray detectors on board NuStar (launched in 2012) or ASTRO-H (scheduled for launch in 2014) will reach to SNe Ia at $\sim 15 \mathrm{Mpc}$, i.e., one SN every few years. Furthermore, according to the present results, the soft $\gamma$-ray detector on board ASTRO-H will be able to detect the $158 \mathrm{keV}$ line emission up to $\sim 25 \mathrm{Mpc}$, i.e., a few SNe Ia per year. Proposed next-generation $\gamma$-ray missions, e.g., GRIPS, could reach to SNe Ia at $\sim 20-35 \mathrm{Mpc}$ by MeV observations. Those would provide new diagnostics and strong constraints on explosion models, detecting rather directly the main energy source of supernova light.
\end{abstract}

Key words: nuclear reactions, nucleosynthesis, abundances - radiative transfer - supernovae: general supernovae: individual (SN 2011fe)

Online-only material: color figures

\section{INTRODUCTION}

It is widely accepted that Type Ia supernovae (SNe Ia) are a major source of $\mathrm{Fe}$ in the universe (see, e.g., Hillebrandt \& Niemeyer 2000 for a review). The thermonuclear explosion of a white dwarf produces ${ }^{56} \mathrm{Ni}$ as a main product (e.g., Nomoto et al. 1984). It decays into ${ }^{56} \mathrm{Co}$ (with an $e$-folding time of $\sim 8.8$ days) and then into ${ }^{56} \mathrm{Fe}(\sim 113$ days). The transitions typically occur into excited states of the daughter nuclei, which generally de-excite by emissions of $\gamma$-rays with characteristic energies of $\sim 1 \mathrm{MeV}$. These $\gamma$-rays energize the thermal electron pool mainly through Compton scattering, which ultimately leads to the optical appearance of SNe Ia.

Although this scenario has been supported by studying optical spectra and light curves, the most direct evidence is still missing, i.e., the detection of the decay $\gamma$-rays and related high energy emissions (e.g., Clayton et al. 1969; Ambwani \& Sutherland 1988; Milne et al. 2004). A marginal detection for the peculiar SN Ia 1991T was reported (Lichti et al. 1994; Morris et al. 1997), while for the closer SN Ia 1998bu only upper limits were obtained (Georgii et al. 2002). The recently discovered SN Ia $2011 \mathrm{fe}$ in the nearby galaxy M101 at $\sim 6.4 \mathrm{Mpc}$ (Nugent et al. 2011a, 2011b) has been observed by INTEGRAL, but only an upper limit has been placed (Isern et al. 2011a, 2011b).
Despite the importance of multi-dimensional structures in state-of-the-art explosion models (e.g., Gamezo et al. 2003; Röpke \& Hillebrandt 2005; Bravo \& García-Senz 2006; Röpke \& Niemeyer 2007; Röpke et al. 2007; Jordan et al. 2008; Seitenzahl et al. 2011) up to now most theoretical studies of the high energy emission of SNe Ia have been restricted to onedimensional models (e.g., Ambwani \& Sutherland 1988; Höflich et al. 1992, 1998; Gómez-Gomar et al. 1998). For a review of the (one-dimensional) theoretical studies of high energy signals from SNe Ia, see Milne et al. (2004). Only recently have the first multi-dimensional studies become available (e.g., Höflich 2002; Hungerford et al. 2003; Maeda 2006; Sim \& Mazzali 2008; Kromer et al. 2010). Höflich (2002) discussed effects of the multi-dimensionality in the explosion (for a few delayed-detonation models) on the line profiles of the 812 and $847 \mathrm{keV}$ lines. Sim \& Mazzali (2008) examined flux evolution in different energy bands based on kinematic models. Kromer et al. (2010) presented a prediction of the high energy signal based on the double-detonation sub-Chandrasekhar models. In this paper, we report the first study on expected high energy emission signatures and their flux evolution from a series of two-dimensional delayed-detonation models.

We use these models to discuss possible constraints on the explosion mechanism of $\mathrm{SNe}$ Ia through the $\gamma$-ray 


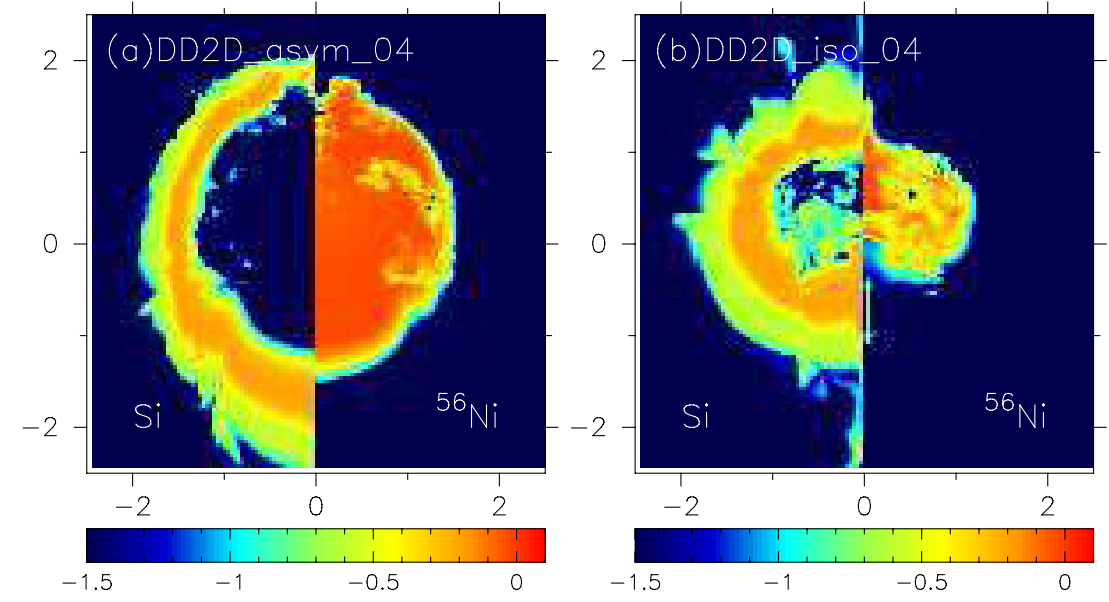

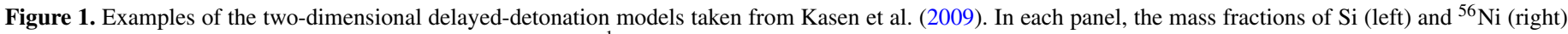
are shown on a logarithmic scale. The axes are in $10,000 \mathrm{~km} \mathrm{~s}^{-1}$.

(A color version of this figure is available in the online journal.)

non-detection from SN 2011 fe by the currently operating instrument SPI on board INTEGRAL (a narrow-line sensitivity of $3.1 \times 10^{-5}$ photons $\mathrm{cm}^{-2} \mathrm{~s}^{-1}$ at $1 \mathrm{MeV}$ for $10^{6} \mathrm{~s}$ exposure; Roques et al. 2003). ${ }^{12}$ We then examine the detectability of the high energy emission from SNe Ia by near-future observatories. These include NuStar (Koglin et al. 2005), Hard X-ray Imager (HXI; Kokubun et al. 2010) and Soft Gamma-ray Detector (SGD; Tajima et al. 2010) on board ASTRO-H (Takahashi et al. 2010), and GRIPS (Greiner et al. 2012). NuStar and HXI are designed to reach to a sensitivity of a few $10^{-8} \mathrm{~cm}^{-2} \mathrm{~s}^{-1} \mathrm{keV}^{-1}$ (for $10^{6} \mathrm{~s}$ ) in the hard X-ray range. The SGD's designed sensitivity is (5-10) $\times 10^{-8} \mathrm{~cm}^{-2} \mathrm{~s}^{-1} \mathrm{keV}^{-1}$ (for $10^{6} \mathrm{~s}$ ) in the soft $\gamma$-ray range below $\sim 600 \mathrm{keV}$. GRIPS is one example of proposed next-generation telescopes, designed to be a factor of $\sim 15$ better in sensitivity than INTEGRAL in the MeV range.

\section{METHODS AND MODELS}

We have performed radiation transfer calculations for the series of two-dimensional delayed-detonation models of Kasen et al. (2009, see also Maeda et al. 2010b; Figure 1). In these models, the thermonuclear explosion was initiated in a number of randomly placed sparks near the center of the white dwarf. The combustion front initially propagates as a subsonic deflagration and later turns into a supersonic detonation (Khokhlov 1991). This scenario involves a deflagration-todetonation transition, which in our models is parameterized in terms of the turbulence strength required at the transition spot. Since the microphysics of deflagration-to-detonation transitions remains an open question, Kasen et al. (2009) suggested a set of criteria that initialize the transition at different Karlovitz numbers. Here, we consider models employing two of these criteria, namely the sets $\mathrm{dc} 2$ and $\mathrm{dc} 3$. The latter requires a larger Karlovitz number and thus delays the transition. Consequently, the detonation proceeds at lower densities and the resulting explosion is typically fainter. Predicted optical emission properties from these models are generally in good agreement with observational properties of normal SNe Ia (Kasen et al. 2009; Maeda et al. 2010a), while those with the largest asymmetry have some problems (Maeda et al. 2010c;

${ }^{12}$ We adopt the sensitivity of SPI from the latest SPI observer's manual at http://www.rssd.esa.int/
Blondin et al. 2011). The simulations were done for the $\mathrm{C}+\mathrm{O}$ white dwarf at the solar metallicity. The detailed nucleosynthesis was followed for a few selected models, and the result was used to interpolate the elemental distribution for $\mathrm{C}, \mathrm{O}, \mathrm{Na}$, $\mathrm{Mg}, \mathrm{Si}, \mathrm{S}, \mathrm{Ar}, \mathrm{Ca}, \mathrm{Ti}, \mathrm{Cr}, \mathrm{Fe}, \mathrm{Co}$, and $\mathrm{Ni}$ in the other models. Since the explosive nucleosynthesis is well characterized by peak temperature and density at the passage of the flame, this procedure would not introduce a large error in the abundance pattern. For each case, 16 different distributions for the initial thermonuclear sparks were investigated. These are divided into two sequences, "iso" and "asym" (named either DD2D_iso_AA or DD2D_asym_AA, with AA spanning from 01 to 08). In the "iso" models, the ignition points were randomly distributed in a sphere around the white dwarf center, while in the "asym" models the ignition points were preferentially offset in a certain direction. The resulting mass of ${ }^{56} \mathrm{Ni}$ ranges from $0.34 M_{\odot}$ to $1.15 M_{\odot}$ (see Table 1 for examples). In the "iso" models, the number of ignited sparks is larger for a larger value of AA. In the "asym" models, the ignited sparks were put in a narrower cone for larger AA with a smaller number of sparks. Generally, a smaller number of initial sparks (smaller AA in "iso" and larger AA in "asym") leads to a weaker deflagration and a stronger detonation, resulting in larger $M\left({ }^{56} \mathrm{Ni}\right)$. We have also investigated the one-dimensional pure deflagration model W7 (Nomoto et al. 1984). In total, 33 models have been investigated.

Transfer of $\gamma$-rays from the decay chain ${ }^{56} \mathrm{Ni} \rightarrow{ }^{56} \mathrm{Co} \rightarrow{ }^{56} \mathrm{Fe}$ has been followed using the three-dimensional time-dependent radiation transfer code of Maeda (2006, see also Kasen et al. 2006; Sim \& Mazzali 2008). In our calculations, Compton scattering, pair creation, and photoelectric absorption have been included as interaction processes. For Compton scattering, the Klein-Nishina cross section is used. For pair production, cross sections are adopted from Hubbell (1969). For photoelectric absorption, cross sections compiled by Höflich et al. (1992) from Veigele (1973) are used, with the same interpolation scheme adopted by Höflich et al. (1992). A test calculation for the transfer code using the W7 model shows a good match to the spectral sequence obtained by a majority of the transfer codes (e.g., Hungerford et al. 2003) presented in Milne et al. (2004) over the energy range between $80 \mathrm{keV}$ and $2 \mathrm{MeV}$ for which the synthetic spectra for the comparison are available in Milne et al. (2004). We are thus confident that our prediction is solid in the soft $\gamma$-ray energy. Milne et al. (2004) noted that there are differences 

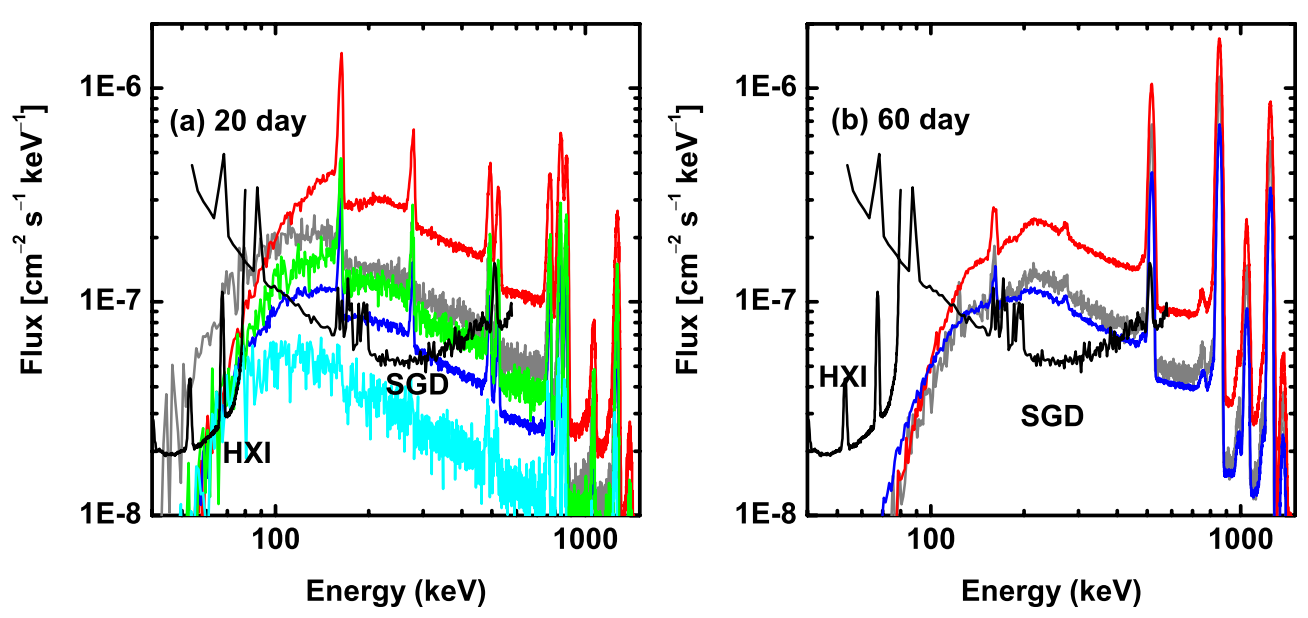

Figure 2. Examples of synthetic spectra at (a) 20 days and (b) 60 days after the explosion. Shown here are angle-averaged spectra for models DD2D_asym_04 (dc2; red line), W7 (gray), and DD2D_iso_04 (dc3; dark blue). The masses of ${ }^{56} \mathrm{Ni}$ are $1.02 M_{\odot}, 0.64 M_{\odot}$, and $0.42 M_{\odot}$, respectively (Table 1). The distance is assumed to be $10 \mathrm{Mpc}$. The angle-dependent spectra seen from two opposite directions are shown for DD2D_iso_04 (green and cyan) at 20 days. At 60 days, the angle dependence is small and thus not shown here. The angle dependence is small for DD2D_asym 04 at both epochs. Sensitivity curves for an exposure with $10^{6}$ s of HXI and SGD on board ASTRO-H (as presented in Tajima et al. 2010; Takahashi et al. 2010) are shown by black lines.

(A color version of this figure is available in the online journal.)

Table 1

Expected Detectability ${ }^{\mathrm{a}}$

\begin{tabular}{|c|c|c|c|c|}
\hline & $M\left({ }^{56} \mathrm{Ni}\right) / M_{\odot}$ & $\begin{array}{c}\text { DD2D_asym_04 } \\
1.02\end{array}$ & $\begin{array}{c}\text { W7 } \\
0.64\end{array}$ & $\begin{array}{c}\text { DD2D_iso_04 } \\
0.42\end{array}$ \\
\hline Band $(\mathrm{keV})$ & Instrument & $\mathrm{Mpc}\left(\mathrm{SNe} \text { year }^{-1}\right)^{\mathrm{b}}$ & & \\
\hline \multirow[t]{3}{*}{$60-80$} & HXI & $13.9(0.43)$ & $17.7(0.96)$ & $10.5(0.09)$ \\
\hline & NuStar (cons.) $)^{\mathrm{c}}$ & $13.0(0.43)$ & $16.5(0.70)$ & $9.7(0.09)$ \\
\hline & NuStar (opt.) ${ }^{\mathrm{c}}$ & $18.4(1.13)$ & $23.3(2.52)$ & $13.8(0.43)$ \\
\hline \multirow[t]{3}{*}{158} & SPI & $4.6(<0.09)$ & $2.9(<0.09)$ & $2.3(<0.09)$ \\
\hline & SGD (cons. $)^{\mathrm{d}}$ & $22.2(2.09)$ & $14.2(0.43)$ & $11.4(0.09)$ \\
\hline & SGD (opt. $)^{\mathrm{d}}$ & $38.5(6.70)$ & $24.6(2.96)$ & $19.7(1.57)$ \\
\hline \multirow[t]{3}{*}{$200-460$} & SPI & $3.7(<0.09)$ & $2.7(<0.09)$ & $2.3(<0.09)$ \\
\hline & SGD (cons.) & $11.6(0.09)$ & $8.6(0.09)$ & $7.1(0.09)$ \\
\hline & SGD (opt.) & $20.2(1.74)$ & $14.8(0.43)$ & $12.3(0.26)$ \\
\hline \multirow[t]{2}{*}{812} & SPI & $4.3(<0.09)$ & $2.6(<0.09)$ & $2.0(<0.09)$ \\
\hline & GRIPS & $16.8(0.87)$ & $10.0(0.09)$ & $7.6(0.09)$ \\
\hline \multirow[t]{2}{*}{847} & SPI & $7.7(0.09)$ & $5.4(<0.09)$ & $4.6(<0.09)$ \\
\hline & GRIPS & $29.8(4.52)$ & $21.0(2.00)$ & $18.0(1.04)$ \\
\hline
\end{tabular}

Notes.

${ }^{a}$ For an exposure of $10^{6} \mathrm{~s}$ centered at the peak flux.

${ }^{\mathrm{b}}$ Limiting distance (expected number of SNe per year), computed for the angleaveraged synthetic spectra. The number of SNe is estimated from SNe Ia at a redshift below 0.01 (thus roughly complete below $40 \mathrm{Mpc}$ ) since 2000 taken from the Asiago Supernova Catalog that is constantly updated with new SN discoveries (Barbon et al. 1999).

${ }^{\mathrm{c}}$ Conservative and optimistic estimates assume 4 and $2 \times 10^{-8} \mathrm{~cm}^{-2} \mathrm{~s}^{-1} \mathrm{keV}^{-1}$, respectively, for the NuStar sensitivity (Koglin et al. 2005).

$\mathrm{d}$ The optimistic estimate assumes the designed sensitivity curve of SGD as of 2010 (Tajima et al. 2010; Takahashi et al. 2010). Since the instrument design may change, we also show a conservative estimate, where we degrade the 2010 sensitivity curve by a factor of three.

in the energy range below $100 \mathrm{keV}$ depending on the detail of the transfer schemes- the variation in the predicted flux just below $80 \mathrm{keV}$ is likely less than $50 \%$ (see Figures 4 and 5 of Milne et al. 2004), but one should keep in mind that this is the level of uncertainty involved in the predicted hard X-ray flux. We assume direct annihilation for the $511 \mathrm{keV}$ lines without positronium formation. This will affect the $511 \mathrm{keV}$ line flux to some extent, but the effect of the positronium formation on the downscattered continuum is not important; the positronium decay continuum emission is concentrated just below $511 \mathrm{keV}$ and the Compton scattering cross section in this energy range is almost identical to that at $511 \mathrm{keV}$. Indeed, Milne et al. (2004) found that there is very little difference between the results from codes that include a positronium continuum component and those that do not.

The models have been mapped onto $129^{3}$ spatial grids. In each simulation, we follow $10^{9}$ photon packets with continuous changes in the spatial position, direction, and spectral energy and energy content. Once they escape from the ejecta they are binned into 10 angular bins, 3000 spectral energy bins, and 36 logarithmically spaced time bins from 5 to 300 days after the explosion. This yields time- and angle-dependent spectra, from which light curves are extracted for specific energy bands.

\section{RESULTS}

\subsection{Spectra}

Figure 2 shows examples of synthetic spectra. The flux is normalized to a distance of $10 \mathrm{Mpc}$. Also shown are designed sensitivity curves of HXI and SGD on board the planned ASTRO-H (Kokubun et al. 2010; Takahashi et al. 2010; Tajima et al. 2010).

The $\gamma$-ray lines are produced by radioactive decays (including the $158 \mathrm{keV}$ and $812 \mathrm{keV}$ lines from ${ }^{56} \mathrm{Ni}$ decay and the $847 \mathrm{keV}$ line from ${ }^{56} \mathrm{Co}$ decay). The lines are Doppler shifted by the bulk expansion. Initially only emission from the outer, thin layer along the line of sight is seen as a (narrow) blueshifted line. As time goes by, the escaping lines become broader and the line center moves to lower energy, following the increasing transparency in the deeper, slower part of the ejecta (resulting in the thicker layer probed in the lines; thus the lines become broader). Compton scattering produces the continuum below the line energies, with the low energy cutoff created by photoelectric absorption. The cutoff energy becomes higher as time goes by due to the increasing contribution to the emission from the deeper part where the mean atomic number and photoelectric cross sections are larger.

At 20 days after the explosion, a greater dependence on the viewing direction is seen in DD2D_iso_04 than in 
DD2D_asym_04, despite the initial large asymmetry in the ignition in the latter. Generally, fainter models with smaller $M\left({ }^{56} \mathrm{Ni}\right)$ show a larger viewing-angle dependence, and this is a specific model prediction for the asymmetric delayed-detonation models (Kasen et al. 2009; Maeda et al. 2010b). Even if the initial asymmetry is large, a strong detonation tends to decrease the inhomogeneity in the final abundance distribution. Faint models with a weak detonation preserve the inhomogeneity in the distribution of ${ }^{56} \mathrm{Ni}$ either created by the initial asymmetry and/or mixing in the deflagration phase (Kasen et al. 2009; Figure 1).

At 20 days, the two-dimensional delayed-detonation models show harder spectra than the W7 model. This is a result of more extended burning and larger photoelectric absorption cross sections near the surface in the two-dimensional models. For example, in the W7 model the mass fraction of Si exceeds 0.1 up to $15,000 \mathrm{~km} \mathrm{~s}^{-1}$ (with the inner boundary of $\sim 10,000 \mathrm{~km} \mathrm{~s}^{-1}$ ) (Nomoto et al. 1984), while in the DD2D_iso_04 model it exceeds 0.1 up to $\sim 14,000-20,000 \mathrm{~km} \mathrm{~s}^{-1}$ depending on the viewing direction despite smaller $M\left({ }^{56} \mathrm{Ni}\right)$ (Figure 1). Models with a strong viewing-angle effect (i.e., faint models) show a harder X-ray cutoff when observed from a brighter line of sight. The large flux is created by a larger amount of ${ }^{56} \mathrm{Ni}$ near the surface in this direction. This, together with other burning products like $\mathrm{Si}$, also increases the photoelectric absorption cross section in the same direction. At 60 days, the optical depth decreases and the viewing-angle effect becomes weak (see also Sim \& Mazzali 2008).

\subsection{Light Curves}

Figure 3 shows light curves for selected energy bands and lines. Figure 4 shows examples of evolution in ratios between two different bands. Figure 5 shows selected continuum and line fluxes for the reference distance of $10 \mathrm{Mpc}$, for all the models and viewing directions, as a function of $M\left({ }^{56} \mathrm{Ni}\right)$. Figure 6 provides a constraint on the nature of SN $2011 \mathrm{fe}$ - the predicted flux in the energy range $830-875 \mathrm{keV}$ at $6.4 \mathrm{Mpc}$. The $158 \mathrm{keV}$ line strength has been extracted from the synthetic spectrum in the energy range $150-168 \mathrm{keV}$. The $812 \mathrm{keV}$ and $847 \mathrm{keV}$ line strengths have been extracted as follows. First, we integrate the synthetic spectra in the energy range $790-900 \mathrm{keV}$, then the total flux is divided into the two lines according to the decay probabilities at each epoch (thus assuming the same cross section for these two lines, which should be a good approximation).

The sensitivities of HXI and SGD are calculated by integrating the designed sensitivity curves (Kokubun et al. 2010; Takahashi et al. 2010; Tajima et al. 2010) in the corresponding energy ranges. For lines, we have integrated the sensitivity curve within the typical FWHM predicted by the models, which evolves from $v / c \sim 0.02$ to 0.035 from 20 to 80 days. We note that for a detailed comparison between the models and observations, differences in the line width need to be taken into account-because of the larger line width and a larger background contamination in brighter models, these models are harder to detect than the first-order estimate provided here (the opposite is true for the fainter models). For NuStar, we have assumed a constant sensitivity across the energy band: 4 (conservative) and 2 (optimistic) $\times 10^{-8} \mathrm{~cm}^{-2} \mathrm{~s}^{-1} \mathrm{keV}^{-1}$ (Koglin et al. 2005). For SPI, the narrow-line sensitivity is multiplied by the degradation factor from the line broadening, which we assume is $\sqrt{\mathrm{FWHM} / \Delta E}$ with a typical resolution $\Delta E=2 \mathrm{keV}$. The degradation factor we adopt is therefore $\sim 1-4$, depending on the energy range and the epoch. It would be possible to re-
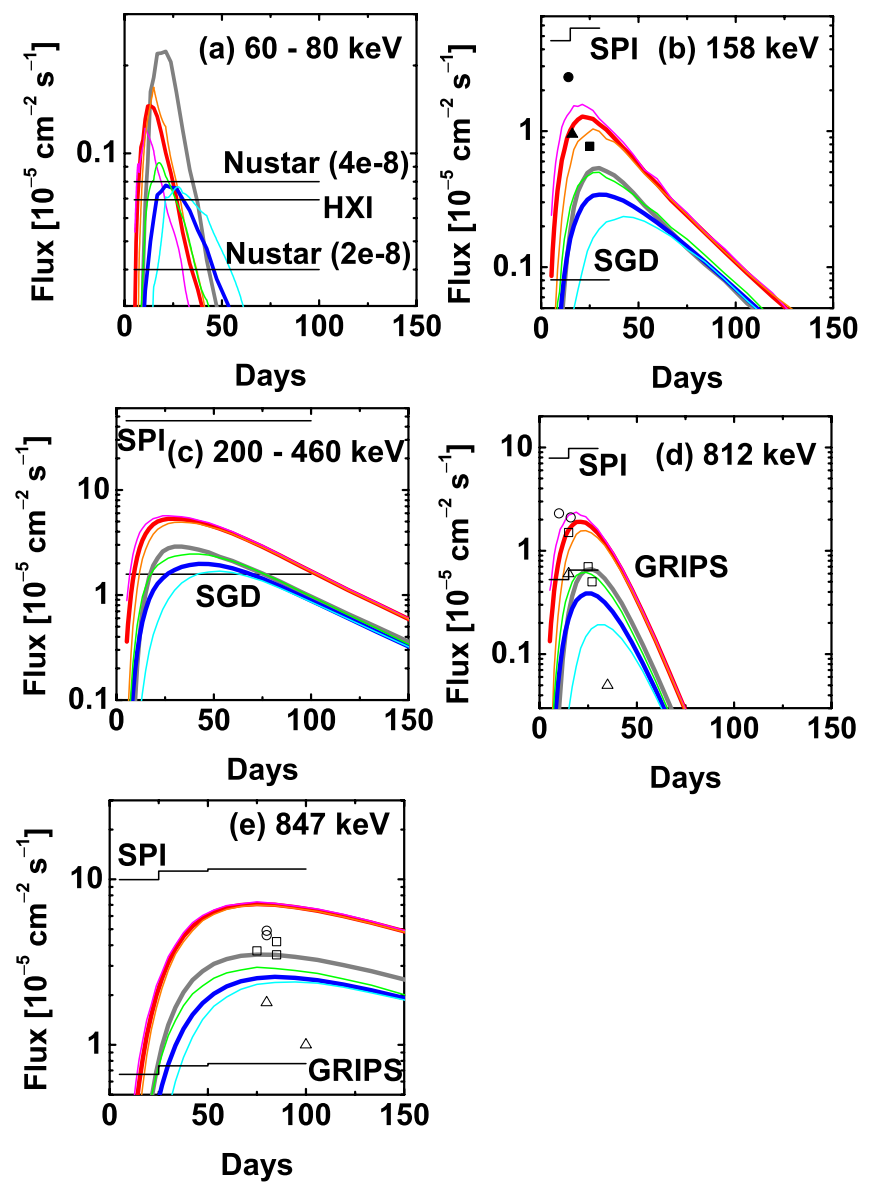

Figure 3. Examples of synthetic light curves for (a) hard X-ray continuum, (b) $158 \mathrm{keV}$ line, (c) integrated flux in $200-460 \mathrm{keV}$, (d) $812 \mathrm{keV}$ line, and (e) $847 \mathrm{keV}$ line. The distance is assumed to be $10 \mathrm{Mpc}$, and the flux is normalized at $10^{-5} \mathrm{~cm}^{-2} \mathrm{~s}^{-1}$. Shown here are light curves for W7 (thick gray), angle-averaged (thick red) and angle-dependent (thin magenta and orange) light curves for DD2D_asym_04 (dc2), angle-averaged (thick blue) and angledependent (thin green and cyan) light curves for DD2D_iso_04 (dc3). For the angle-dependent curves, two opposite directions are shown. For comparison, we show the predictions for the peak days and peak fluxes for other model variants: for the $158 \mathrm{keV}$ flux from Gómez-Gomar et al. (1998) and for the 812 and $847 \mathrm{keV}$ fluxes from Milne et al. (2004). For the $158 \mathrm{keV}$ case, the following three models are shown: a pure detonation model DET (filled circle), a delayed-detonation model DEL (filled square), and a sub-Chandrasekhar model SUB (filled triangle). For the $812 \mathrm{keV}$ and $847 \mathrm{keV}$ cases, the following models are shown: "luminous" SN Ia models HECD and W7DT (open circles), "normal-brightness" models HED8, DD202c, and W7 (open squares), and "subluminous" models HED6 and PDD54 (open triangles). Sensitivity curves (with an exposure of $10^{6} \mathrm{~s}$ ) for several current and future instruments are shown by black lines.

(A color version of this figure is available in the online journal.)

duce the resulting degradation when background features are well constrained and stable, and/or when specific line shapes can be assumed or tested. For GRIPS, we simply assume that the narrow-line sensitivity is better than SPI by a factor of 15 (Greiner et al. 2012).

In the hard X-ray continuum, the two-dimensional delayeddetonation models peak fainter than the W7 model, even if $M\left({ }^{56} \mathrm{Ni}\right)$ is larger, as a result of the larger mean atomic number (due to more complete burning) and larger photoelectric absorption cross sections near the surface. The signal can therefore be a strong diagnostic for the composition near the surface and the mode of the flame propagation. The models with larger $M\left({ }^{56} \mathrm{Ni}\right)$ peak earlier, at a higher flux level: thus the hard X-ray alone (as well as the $158 \mathrm{keV}$ line discussed 

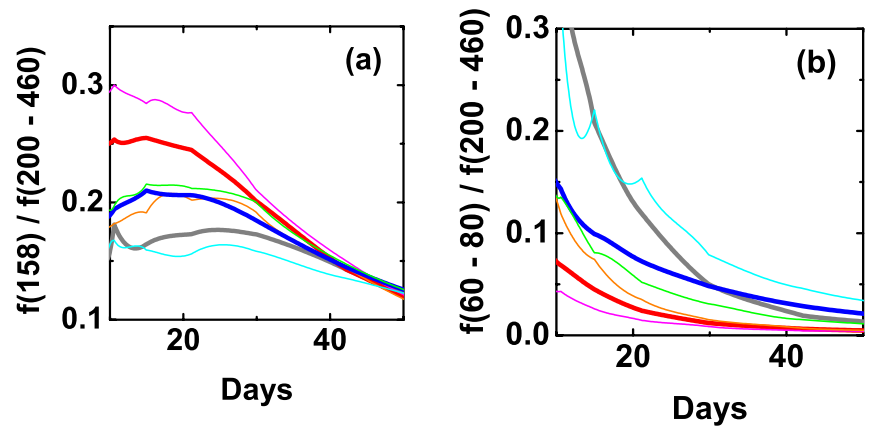

Figure 4. Examples of the flux ratio diagnostics: (a) the ratio between the $158 \mathrm{keV}$ line and the $200-460 \mathrm{keV}$ continuum and (b) the ratio between the $60-80 \mathrm{keV}$ continuum and the $200-460 \mathrm{keV}$ continuum. Shown here are model curves for W7 (thick gray), angle-averaged (thick red) and angle-dependent (thin magenta and orange) curves for DD2D_asym_04 (dc2), angle-averaged (thick blue) and angle-dependent (thin green and cyan) curves for DD2D_iso_04 (dc3). For the angle-dependent curves, two opposite directions are shown.

(A color version of this figure is available in the online journal.)

below) could give a rough constraint on $M\left({ }^{56} \mathrm{Ni}\right)$ independently from optical observations. The two-dimensional models show a strong angle dependence. The angle variation in the model flux reaches nearly $100 \%$ for models with $M\left({ }^{56} \mathrm{Ni}\right) \sim 0.6 M_{\odot}$, while for the brighter models it is at most $\sim 40 \%$ (Figure 5). If an SN Ia as close as SN 2011fe should appear again in the coming decade when the near-future hard X-ray instruments (HXI and NuStar) will be operating, the signal should be detectable by these instruments.

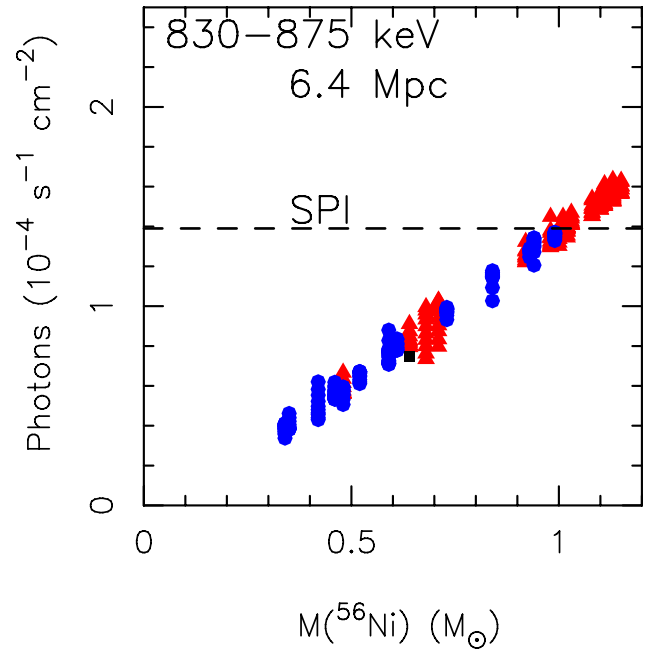

Figure 6. Constraint on the models from the INTEGRAL/SPI observation of SN $2011 \mathrm{fe}$. The model fluxes for an SN at $6.4 \mathrm{Mpc}$ are time-averaged within days 45-88. The "asym" models are shown by red triangles, "iso" models by blue circles, and W7 by a black square. The $2 \sigma$ upper limit obtained by SPI (Isern et al. 2011a) is shown by the dashed line.

(A color version of this figure is available in the online journal.)

The behavior of the $158 \mathrm{keV}$ line $\left({ }^{56} \mathrm{Ni} \rightarrow{ }^{56} \mathrm{Co}\right)$ is similar to that of the hard X-ray continuum (see above), except that the difference between the two-dimensional models and W7 is not large for given $M\left({ }^{56} \mathrm{Ni}\right)$, since the dominant interaction
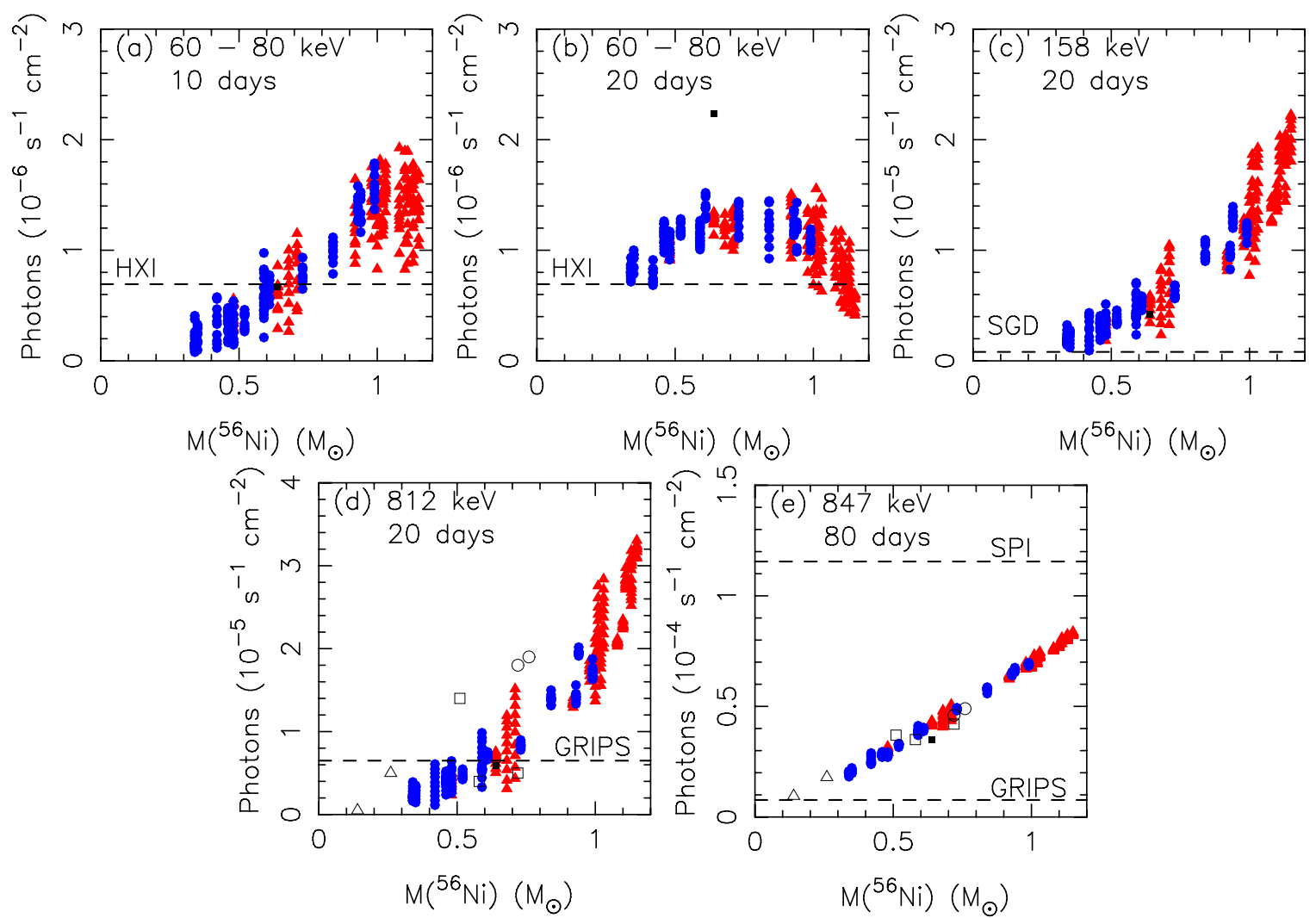

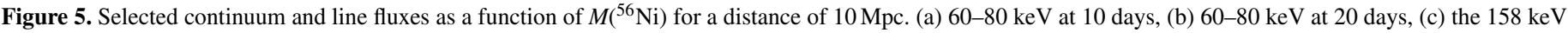

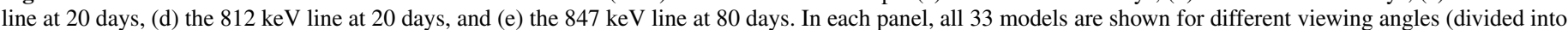

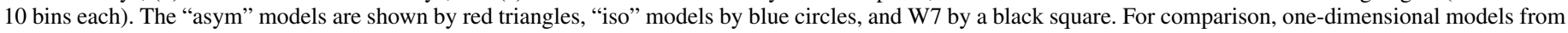

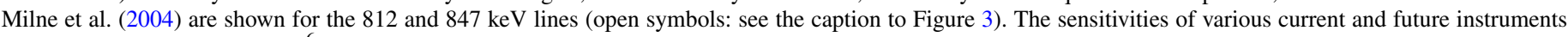
are shown for an exposure of $10^{6} \mathrm{~s}$.

(A color version of this figure is available in the online journal.) 
process is Compton scattering and the difference in the surface composition is not important. Not many previous theoretical studies have explicitly addressed the $158 \mathrm{keV}$ line strength-in Figure 3 we plot the prediction on the peak $158 \mathrm{keV}$ flux from Gómez-Gomar et al. (1998) together with our two-dimensional delayed-detonation model predictions. The models of GómezGomar et al. (1998) are one-dimensional models, including a delayed-detonation model $\left(M\left({ }^{56} \mathrm{Ni}\right)=0.8 M_{\odot}\right)$, a pure detonation model $\left(M\left({ }^{56} \mathrm{Ni}\right)=0.7 M_{\odot}\right)$, and a sub-Chandrasekhar explosion model $\left(M\left({ }^{56} \mathrm{Ni}\right)=0.6 M_{\odot}\right)$. In Gómez-Gomar et al. (1998), the $158 \mathrm{keV}$ flux was extracted from an energy range corresponding to $\sim 1.2 \times$ FWHM. Thus, their line fluxes were extracted from a narrower range than what we adopt for our models. The difference should not be large as we deal with a line. Their delayed-detonation model follows our (angle-averaged) model behavior. The pure detonation model predicts a larger peak flux than any of the two-dimensional delayed-detonation models, and the difference is about a factor of three for the same $M\left({ }^{56} \mathrm{Ni}\right)$. The sub-Chandrasekhar model peaks earlier than for the delayed-detonation model, and the peak flux is higher by about a factor of two than the corresponding delayeddetonation model with similar $M\left({ }^{56} \mathrm{Ni}\right)$. All of our delayeddetonation models, as well as other typical models (e.g., GómezGomar et al. 1998), are reachable by SGD for an SN at $10 \mathrm{Mpc}$ (Figure 3).

The $158 \mathrm{keV}$ line is generally the strongest signal in the first month. Since the expected behavior is sensitive to the distribution of ${ }^{56} \mathrm{Ni}$, it is most useful to distinguish models which result in a very different spatial distribution of ${ }^{56} \mathrm{Ni}$. For $\mathrm{SN}$ $2011 \mathrm{fe}$, the $2 \sigma$ upper limit on the integrated flux in the energy range $160-166 \mathrm{keV}$ at $\sim 10$ days after the explosion has been placed at $7.5 \times 10^{-5}$ photons $\mathrm{cm}^{-2} \mathrm{~s}^{-1}$ by the SPI observation with an exposure of $\sim 10^{6} \mathrm{~s}$ (Isern et al. 2011b). This upper limit rejects none of our models and neither of the other models shown in Figure 3-our model fluxes extracted in the energy range $150-168 \mathrm{keV}$ are lower than $2 \times 10^{-5}$ photons $\mathrm{cm}^{-2} \mathrm{~s}^{-1}$, and should be even lower for the $160-166 \mathrm{keV}$ range. The pure detonation model in Gómez-Gomar et al. (1998) has the largest flux among the models presented here, $\sim 6 \times$ $10^{-5}$ photons $\mathrm{cm}^{-2} \mathrm{~s}^{-1}$ at the distance of $6.4 \mathrm{Mpc}$ which is still below the observational upper limit.

The light curve of the $200-460 \mathrm{keV}$ range and that of the $812 \mathrm{keV}$ line follow a behavior similar to that of the $158 \mathrm{keV}$ line (see below for more detailed discussion on the 200-460 keV continuum). In Figures 3 and 5, we show the predictions of other well-investigated models together with our two-dimensional delayed-detonation models for the $812 \mathrm{keV}$ line flux. These model predictions have been taken from Milne et al. (2004): normal-brightness SN Ia models (HED8, DD202c, W7), luminous models (HECD, W7DT), and subluminous models (HED6, PDD54) covering a wide range of $M\left({ }^{56} \mathrm{Ni}\right), 0.14-0.76 M_{\odot}$. The models which have a large amount of ${ }^{56} \mathrm{Ni}$ near the surface peak earlier and are brighter than our two-dimensional delayed-detonation model sequence for given $M\left({ }^{56} \mathrm{Ni}\right)$. It is seen that the typical behavior of the twodimensional delayed-detonation model is similar to the onedimensional deflagration model W7 and the one-dimensional delayed-detonation model DD202c, except that a variation in the flux is expected for different viewing directions in the twodimensional models (like in the hard X-ray continuum and the $158 \mathrm{keV}$ line). We note that Milne et al. (2004) integrated the flux for the 812 and $847 \mathrm{keV}$ lines in the energy range $810-885 \mathrm{keV}$, narrower than our definition $(790-900 \mathrm{keV})$. However, the continuum level is quite low, and thus the difference is negligible (i.e., at most a few percent).

The $847 \mathrm{keV}$ line $\left({ }^{56} \mathrm{Co} \rightarrow{ }^{56} \mathrm{Fe}\right)$ is the strongest line for $\mathrm{SNe}$ Ia and it peaks around 60-100 days after the explosion. This line is insensitive in its behavior to the viewing direction due to a small optical depth at these late epochs. Indeed, the behavior of this line is rather insensitive to model variants as well, but mostly determined by $M\left({ }^{56} \mathrm{Ni}\right)$, up to $\sim 30 \%$ level, as shown in Figure 5. This means that the detection of this signal around the peak would not discriminate between different model variants (such as pure detonation, delayed-detonation, one-dimensional or multi-D), but provides a direct estimate of $M\left({ }^{56} \mathrm{Ni}\right)$ where the uncertainty would come mostly from the limited measurement accuracy rather than from the model uncertainties.

A constraint from the reported upper limit on the $\gamma$-ray signal from SN 2011fe by SPI/INTEGRAL (Isern et al. 2011a) is the following. With $\sim 3 \times 10^{6}$ s exposure at days $45-88$, they placed a preliminary $95 \%$ upper limit $(2 \sigma)$ of $1.39 \times$ $10^{-4}$ photons cm $\mathrm{cm}^{-2} \mathrm{~s}^{-1}$ in the energy range $830-875 \mathrm{keV}$ and $1.20 \times 10^{-4}$ photons $\mathrm{cm}^{-2} \mathrm{~s}^{-1}$ in $835-870 \mathrm{keV}$. Adopting typical model predictions for the line profile, the former likely includes most of the flux in the $847 \mathrm{keV}$ line, while the latter will miss about $10 \%-15 \%$ of the flux. Thus, the constraints from these different band passes are similar. In Figure 6, we show our model predictions at $6.4 \mathrm{Mpc}$, where the model fluxes are averaged over the SPI observation time window (45-88 days). The reported upper limit rejects the models with $M\left({ }^{56} \mathrm{Ni}\right) \gtrsim 1.0 M_{\odot}$.

The constraint here should be compared with the optical properties of SN 2011fe. The optical light curve evolution suggests that it is a normal SN Ia peaking at $-19.13 \mathrm{mag}$ in $B$ band, indicating that $0.45 M_{\odot} \lesssim M\left({ }^{56} \mathrm{Ni}\right) \lesssim 0.6 M_{\odot}$ has been produced in the explosion (Nugent et al. 2011b; Röpke et al. 2012). Röpke et al. (2012) presented an optical-spectral analysis based on two explosion models, a three-dimensional delayed-detonation model (similar to those presented in this paper) and a white dwarf-white dwarf merger, both models having $M\left({ }^{56} \mathrm{Ni}\right) \sim$ $0.6 M_{\odot}$. Both models show a general agreement with the observed spectral sequence: Röpke et al. (2012) found a slightly better match for the merger model but could not place a clear preference based on the available optical data. The constraint from the SPI observation, $M\left({ }^{56} \mathrm{Ni}\right) \lesssim 1.0 M_{\odot}$, is consistent with the optical emission analysis, but unfortunately not deep enough to provide further constraints. However, we emphasize that the constraint from the high energy emission is fully independent of the optical emission analysis and suffers from far fewer theoretical uncertainties. Thus, it demonstrates that a strong constraint can potentially be obtained from high energy signals for future nearby SNe Ia.

We predict different behaviors for different energy ranges, depending on the models and viewing directions (see above). This provides strong diagnostics on the explosion mechanism. Figure 4 shows two examples: the flux ratio between the $158 \mathrm{keV}$ line and the $200-460 \mathrm{keV}$ continuum, and that between the $60-80 \mathrm{keV}$ continuum and the $200-460 \mathrm{keV}$ continuum. The first example is sensitive to the column mass density above the ${ }^{56} \mathrm{Ni}$-rich region. The dominant interaction process at $158 \mathrm{keV}$ and in the high energy continuum is Compton downscattering (there is a significant contribution from photoelectric absorption at $158 \mathrm{keV}$, but this contribution is negligible in the argument). The strength of the $158 \mathrm{keV}$ line is proportional to the escape fraction of photons, while that of the $200-460 \mathrm{keV}$ continuum is the combination of the scattering fraction of the decay lines and the escape fraction of the downscattered photons. As such, 
the ratio is larger for smaller optical depth due to Compton scattering (i.e., for a smaller amount of material above ${ }^{56} \mathrm{Ni}$ ). It is seen in Figure 4 that models with a large amount of ${ }^{56} \mathrm{Ni}$ and/or those viewed on-axis have a high flux ratio (i.e., a situation in which the distribution of ${ }^{56} \mathrm{Ni}$ is extended toward the surface along the line of sight). The ratio between the hard $\mathrm{X}$-ray continuum and the soft gamma-ray continuum behaves in the opposite way. This is an example of the diagnostics about the chemical composition near the surface. At $60-80 \mathrm{keV}$, photoelectric absorption is the dominant interaction process. As such, the ratio here is a measure of the average atomic number near the surface through the cross section to photoelectric absorption. Models with the largest abundance of Fe-peak elements near the (line-of-sight) surface have the smallest ratio.

\subsection{Detectability}

Table 1 summarizes the expected detectability of a few selected models. The exposure of $10^{6} \mathrm{~s}$ is assumed to be centered on the peak flux date. The time-averaged fluxes extracted from the spectral time series are then compared with the sensitivities of various instruments. In practice, the peak date is not known in advance. This is not a big problem for the $847 \mathrm{keV}$ line, but it matters for the low energy bands, e.g., the hard X-rays and the $158 \mathrm{keV}$ line, where the peak date is sensitive to the model details (see Figure 3). In planning observations, it is thus encouraged to take into account the evolution of the optical emission so that the coordinated observation covers the peak flux. This, however, requires a very early discovery and intensive followup as was done for SN 2011 fe (Nugent et al. 2011b). Table 1 also gives the expected number of SNe Ia per year based on the Asiago Supernova Catalog that is constantly updated with new SN discoveries (Barbon et al. 1999; see Horiuchi \& Beacom 2010 for the local SN Ia rate).

In the well-studied $847 \mathrm{keV}$ line, we estimate that SPI on board INTEGRAL reaches to $\mathrm{SNe}$ of normal brightness at $\sim 6 \mathrm{Mpc}$ in an ideal situation. This can be optimistic, though, as in practice the SPI observation of SN $2011 \mathrm{fe}$ with $3 \times 10^{6} \mathrm{~s}$ did not detect any positive signals for the data analysis performed so far. In our estimate of the SPI detectability, we have not included the sensitivity loss from modeling the background that could further reduce the sensitivity beyond the increased statistical fluctuations when using a broad energy band. This loss of sensitivity depends on the quality of the background modeling in the data analysis, so further analysis of the SPI data would reduce the upper limit below the value reported by Isern et al. (2011a, 2011b). The observation (with the data analysis so far) does constrain $M\left({ }^{56} \mathrm{Ni}\right) \lesssim 1.0 M_{\odot}$ produced in SN $2011 \mathrm{fe}$. A future telescope like GRIPS is estimated to reach to $\sim 20 \mathrm{Mpc}$ in the $847 \mathrm{keV}$ line, if the sensitivity is improved by a factor of 15 compared to SPI as is designed. Since the $847 \mathrm{keV}$ line luminosity evolves slowly around the peak, even an exposure as long as $3 \times 10^{6} \mathrm{~s}$ could be coordinated (as was done for SN $2011 \mathrm{fe}$; Isern et al. 2011a), and then the detection horizon will even extend to $\sim 35 \mathrm{Mpc}$.

We have found that the near-future instruments for hard $\mathrm{X}$-rays and soft $\gamma$-rays, NuStar (launched in 2012 July) and $A S T R O-H$ (launch planned for 2014), will potentially provide detection of the high energy emission from extragalactic SNe Ia almost annually. NuStar and HXI are estimated to reach to $\mathrm{SNe}$ Ia up to $\sim 15 \mathrm{Mpc}$ (for $\mathrm{SNe}$ Ia of normal luminosity), i.e., roughly one SN every few years. The $158 \mathrm{keV}$ line will be detectable by SGD up to a distance of $\sim 25 \mathrm{Mpc}$, i.e., a few SNe per year. The continuum between 200 and $460 \mathrm{keV}$ will be detectable up to $\sim 14 \mathrm{Mpc}$. Even if we make a very conservative assumption that the SGD sensitivity curve would be degraded from the present design by a factor of three, normal SNe Ia at $\sim 15 \mathrm{Mpc}$ will be reachable (Table 1 ). We note that the model prediction is more solid for the $158 \mathrm{keV}$ line than for the hard $\mathrm{X}$-rays; the degree of agreement between different transfer codes (tested for the same model) is worse in the hard X-rays due to more complicated cross sections in this energy range (Milne et al. 2004; see Section 2). The hard X-ray and the $158 \mathrm{keV}$ line evolve relatively quickly, but the flux levels stay almost constant around the peak for $\sim 10-20$ days; thus an exposure of $10^{6} \mathrm{~s}$ is appropriate. It also requires an $\mathrm{SN}$ discovered soon after the explosion. Such an early detection is now feasible thanks to new transient searches with high cadence (e.g., Nugent et al. 2011a, 2011b).

Another possible concern about the hard X-ray band study is contamination from background sources. The predicted peak hard X-ray luminosity from an $\mathrm{SN}$ is $\sim 10^{39} \mathrm{erg} \mathrm{s}^{-1}$. For example, in M101 there are $\sim 100 \mathrm{X}$-ray sources above $\sim 10^{36} \mathrm{erg} \mathrm{s}^{-1}$ in the energy range $0.1-8 \mathrm{keV}$ (Pence et al. 2001). For a conservative angular resolution of 2 arcmin for HXI, about 5 or 10 such sources may be unresolved from the SN emission. The expected total flux from these contaminated sources is, however, far below the predicted SN hard X-ray luminosity. The probability that a host galaxy has a powerful active galactic nucleus and that an $\mathrm{SN}$ is close to the core is small for nearby $\mathrm{SNe}$ (neither is the case for SN 2011fe). Also, the characteristic temporal evolution of the SNe, i.e., the quick decrease of the flux (a magnitude decrease in one or two months) and no repetition, should be distinguishable from underlying unrelated sources.

For $A S T R O-H$, we have performed simulations for the designed detector's response by using some of the synthetic spectra as input. We have used the detector's response and background presented by Tajima et al. (2010) and Takahashi et al. (2010), and this corresponds to the "optimistic" case in Table 1. Figure 7 shows examples of the simulations for the three models (DD2D_asym_04, W7, DD2D_iso_04) at a distance of 5, 15 , and $25 \mathrm{Mpc}$. We have used synthetic spectra at 20 days after the explosion. This is indeed conservative, since only the brightest model (DD2D_asym_04) reaches the peak at 20 days, and the other two models have not yet reached the maximum flux at 20 days. The simulation assumes an exposure of $10^{6} \mathrm{~s}$. For the spectral region around the $158 \mathrm{keV}$ line, all these three models are detectable at $25 \mathrm{Mpc}$, confirming our estimate in Table 1 (the simulation indeed indicates that the values in Table 1 may even be underestimated). Detecting the signal as a "line" is more difficult-for example, at $15 \mathrm{Mpc}$ we can still identify the $158 \mathrm{keV}$ "line" in the spectrum of the DD2D_asym_04 model, but not for the other two models. The simulation also shows that resolving the $158 \mathrm{keV}$ line profiles can be definitely done for $\mathrm{SNe}$ Ia at $5 \mathrm{Mpc}$, but is not possible for $\mathrm{SNe}$ Ia at a distance of $10 \mathrm{Mpc}$ or larger. A slightly worse level of agreement between the simple estimate and the response simulations is found for the HXI.

\section{CONCLUSIONS AND DISCUSSION}

In this paper, we have reported properties of high energy emissions from the radioactive decay chain ${ }^{56} \mathrm{Ni} \rightarrow{ }^{56} \mathrm{Co} \rightarrow{ }^{56} \mathrm{Fe}$ in SNe Ia. A series of two-dimensional delayed-detonation models have been investigated.

We estimate, for the narrow-line sensitivity of $3 \times$ $10^{5}$ photons $\mathrm{cm}^{-2} \mathrm{~s}^{-1}$, that the $847 \mathrm{keV}$ line from the decay of ${ }^{56} \mathrm{Co}$ is detectable by SPI/INTEGRAL for the closest $\mathrm{SNe}$ 

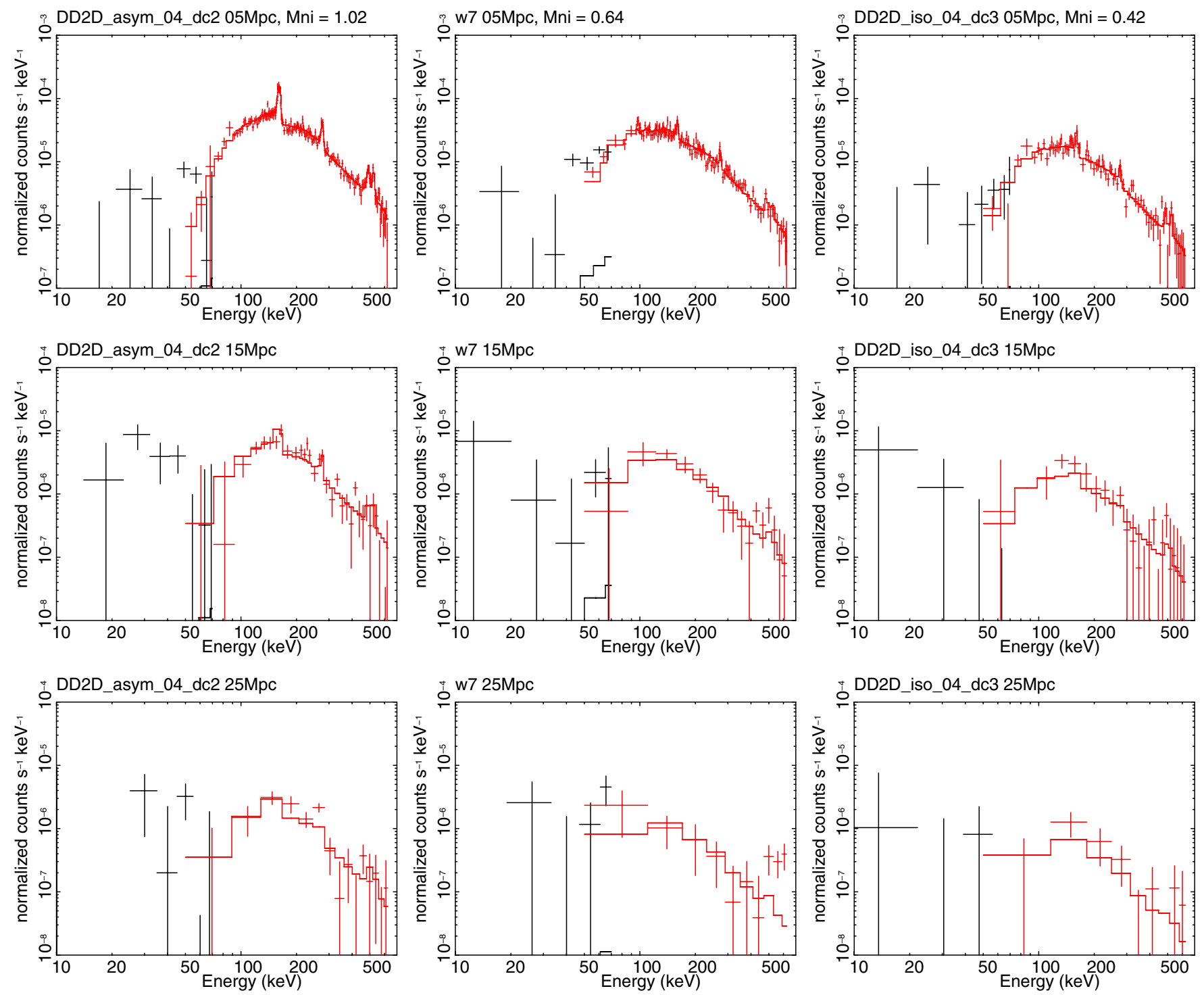

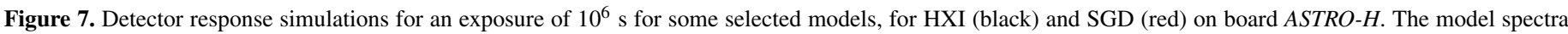

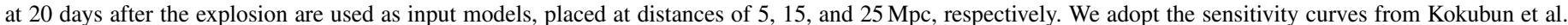
(2010), Tajima et al. (2010), and Takahashi et al. (2010).

(A color version of this figure is available in the online journal.)

within $\sim 6 \mathrm{Mpc}$, at 60 days after the explosion and thereafter. This, however, is likely optimistic in view of the non-detection of the signal from SN 2011 fe by SPI (Section 3.3).

The flux of the $847 \mathrm{keV}$ line is sensitive to $M\left({ }^{56} \mathrm{Ni}\right)$ but not to other model details, such as the progenitor mass, the flame propagation modes, one-dimensional or multi-D, or the viewing direction. Thus, the upper limit of the SPI observation of SN $2011 \mathrm{fe}$ is directly translated to a constraint on the mass of ${ }^{56} \mathrm{Ni}$, as $M\left({ }^{56} \mathrm{Ni}\right) \lesssim 1.0 M_{\odot}$. This is not as strong as the constraint placed by optical emission analysis (Nugent et al. 2011b; Röpke et al. 2012), but is totally independent from and more direct than the optical emission analysis. This shows a potential to place a strong constraint on the nature of the explosion through the high energy emission. In the earlier phase, the most constraining signal is the $158 \mathrm{keV}$ line. The behavior here is sensitive to different models (e.g., the thermonuclear flame modes, initial conditions, and viewing angles within the delayed-detonation scenario) - the feature essentially traces how much material is present atop of the ${ }^{56} \mathrm{Ni}$-rich region. SN $2011 \mathrm{fe}$ was observed by INTEGRAL with an exposure of $\sim 10^{6} \mathrm{~s}$ starting at $\sim 5$ days after the discovery (Isern et al. 2011b), but unfortunately the reported upper limit is not deep enough to reject any models presented in this paper (including the pure detonation model). For more detailed and quantitative analysis, variations in the line width predicted for different models will need to be taken into account.

While most previous studies focused on the detectability of the radioactive signals in the $\mathrm{MeV}$ range, we suggest that detecting soft $\gamma$-rays and hard X-rays is more promising with the new, near-future observatories (ASTRO-H and NuStar). We have found that the $158 \mathrm{keV}$ line is detectable up to $\sim 25 \mathrm{Mpc}$ with SGD on board $A S T R O-H$, and the hard X-ray continuum up to $\sim 15 \mathrm{Mpc}$ with HXI on board ASTRO-H and NuStar. These near-future observatories, which we predict are able to detect the high energy emission almost annually, are expected to provide practically applicable diagnostics on the explosion mechanism.

1. The hard X-ray continuum provides a measure of the composition near the surface.

2. The $158 \mathrm{keV}$ line flux provides a measure of how much material is present above the ${ }^{56} \mathrm{Ni}$-rich region. 
3. Accordingly, some line-to-continuum ratios, shown to be accessible by these new instruments, will provide a strong constraint on the explosion mechanism (or the viewing direction in the two-dimensional delayed-detonation models).

Compared to other model variants (e.g., one-dimensional pure deflagration model, one-dimensional pure detonation model), the two-dimensional delayed-detonation model tends to predict a lower (angle-averaged) flux in these energy ranges. Thus, our estimate on the detectability of these features with the future observatories may well be conservative. The hard $\mathrm{X}$-ray continuum and the $158 \mathrm{keV}$ line data alone could be used to obtain a rough constraint on $M\left({ }^{56} \mathrm{Ni}\right)$, but this is contaminated by the factors arising from different explosion models as described above.

According to the systematic study of the two-dimensional delayed-detonation models, we have the following solid prediction for this model sequence: for a large statistical sample, a possible scatter in the peak flux may limit the degree of the asymmetry in the explosions. We predict larger asymmetry, thus a larger scatter in the peak flux, for fainter SNe Ia according to the delayed-detonation model.

Future new-generation $\gamma$-ray observatories like GRIPS are expected to deepen the observable horizon in the $\mathrm{MeV}$ range up to $\sim 20 \mathrm{Mpc}$, or even to $35 \mathrm{Mpc}$ for an exposure as long as $3 \times 10^{6} \mathrm{~s}$. The $812 \mathrm{keV}$ line can be used in a way similar to the emission features in the softer band as described above. The peak $847 \mathrm{keV}$ line flux alone is a very good tracer for $M\left({ }^{56} \mathrm{Ni}\right)$; for the model sequences we explore in this paper (the two-dimensional delayed-detonation models, one-dimensional models including various flame propagation modes, those based on Chandrasekhar and sub-Chandrasekhar progenitors), it is insensitive to the model variants and the viewing direction, thus being a direct probe of explosive nucleosynthesis in SNe Ia.

The authors thank Stuart Sim for discussion and useful comments on the manuscript. This research is supported by World Premier International Research Center Initiative (WPI Initiative), MEXT, Japan. K.M. acknowledges financial support by a Grant-in-Aid for Scientific Research for Young Scientists (23740141) and by the Max-Plank Society as a short-term visitor. The work of F.R. was supported by the Emmy Noether Program (RO 3676/1-1) of the Deutsche Forschungsgemeinschaft and the ARCHES award.

\section{REFERENCES}

Ambwani, K., \& Sutherland, P. 1988, ApJ, 325, 820

Barbon, R., Buond, V., Cappellaro, E., \& Turatto, M. 1999, A\&AS, 139, 531

Blondin, S., Kasen, D., Röpke, F. K., Kirshner, R. P., \& Mandel, K. S. 2011, MNRAS, 417, 1280

Bravo, E., \& Garcís-Senz, D. 2006, ApJ, 642, L157

Clayton, D. D., Colgate, S.A, \& Fishman, G. J. 1969, ApJ, 155, 75

Gamezo, V. N., Khokhlov, A. M., Oran, E. S., Chtchelkanova, A. Y., \& Rosenberg, R. O. 2003, Science, 299, 77

Georgii, R., Plüschke, S., Diehl, R., et al. 2002, A\&A, 394, 517

Gómez-Gomar, J., Isern, J., \& Jean, P. 1998, MNRAS, 295, 1

Greiner, J., Mannheim, K., Aharonian, F., et al. 2012, Exp. Astron., 34, 551

Hillebrandt, W., \& Niemeyer, J. C. 2000, ARA\&A, 38, 191

Höflich, P. 2002, New Astron. Rev., 46, 475

Höflich, P., Khokhlov, A., \& Müller, E. 1992, A\&A, 259, 549

Höflich, P., Wheeler, J. C., \& Khokhlov, A. 1998, ApJ, 492, 228

Horiuchi, S., \& Beacom, J. F. 2010, ApJ, 723, 329

Hubbell, J. H. 1969, Natl. Standard Ref. Data System, Natl. Bureau of Standards Rep. 29 (Washington, DC: NBS)

Hungerford, A. L., Fryer, C. L., \& Warrenm, M. S. 2003, ApJ, 594, 390

Isern, J., Bravo, E., Knodlseder, J., et al. 2011a, ATel, 3822

Isern, J., Jean, P., Bravo, E., et al. 2011b, ATel, 3683

Jordan, G. C., Fisher, R. T., Townsley, D. M., et al. 2008, ApJ, 681, 1448

Kasen, D., Röpke, F. K., \& Woosley, S. E. 2009, Nature, 460, 869

Kasen, D., Thomas, R. C., \& Nugent, P. 2006, ApJ, 651, 366

Khokhlov, A. 1991, A\&A, 245, 114

Koglin, J. E., Christensen, F. E., Craig, W. W., et al. 2005, Proc. SPIE, 5900, 266

Kokubun, M., Nakazawa, K., Enoto, T., et al. 2010, Proc. SPIE, 7732, 33

Kromer, M., Sim, S., Fink, M., et al. 2010, ApJ, 719, 1067

Lichti, G. G., Bennett, K., den Herder, J. W., et al. 1994, A\&A, 292, 569

Maeda, K. 2006, ApJ, 644, 385

Maeda, K., Benetti, S., Stritzinger, M., et al. 2010a, Nature, 466, 82

Maeda, K., Röpke, F. K., Fink, M., et al. 2010b, ApJ, 712, 624

Maeda, K., Taubenberger, S., Sollerman, J., et al. 2010c, ApJ, 708, 1703

Milne, P. A., Hungerford, A. L., Fryer, C. L., et al. 2004, ApJ, 613, 1101

Morris, D. J., Bennett, K., Bloemen, H., et al. 1997, in AIP Conf. Proc. 410,

The Fourth Compton Symposium, ed. C. D. Dermer, M. S. Strickman, \& J. D. Kurfess (Melville, NY: AIP), 1084

Nomoto, K., Thielemann, F.-K., \& Yokoi, K. 1984, ApJ, 286, 644

Nugent, P., Sullivan, M., Bersier, D., et al. 2011a, ATel, 3581

Nugent, P., Sullivan, M., Cenko, S. B., et al. 2011b, Nature, 480, 344

Pence, W. D., Snowden, S. L., \& Mukai, K. 2001, ApJ, 561, 189

Röpke, F. K., \& Hillebrandt, W. 2005, A\&A, 431, 635

Röpke, F. K., Kromer, M., Seitenzahl, I. R., et al. 2012, ApJ, 750, L19

Röpke, F. K., \& Niemeyer, J. C. 2007, ApJ, 660, 1344

Röpke, F. K., Woosley, S. E., \& Hillebrandt, W. 2007, A\&A, 464, 683

Roques, J. P., Schanne, S., von Kienlin, A., et al. 2003, A\&A, 411, L91

Seitenzahl, I. R., Ciaraldi-Schoolmann, F., \& Röpke, F. K. 2011, MNRAS, 414 , 2709

Sim, S. A., \& Mazzali, P. A. 2008, MNRAS, 385, 1681

Tajima, H., Blandford, R., Enoto, T., et al. 2010, Proc. SPIE, 7732, 34

Takahashi, T., Mitsuda, Kazuhisa, Kelley, R., et al. 2010, Proc. SPIE, 7732, 27

Veigele, W. J. 1973, At. Data, 5, 51 